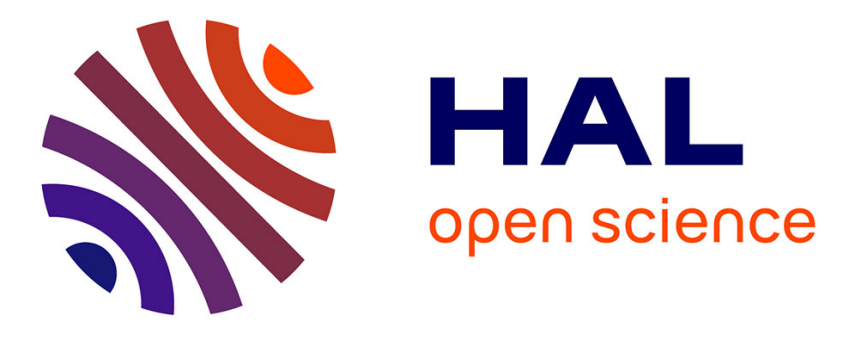

\title{
Fluid-Induced Rotordynamic Forces on a Whirling Centrifugal Pump
}

\author{
Dario Valentini, Giovanni Pace, Angelo Pasini, Lucio Torre, Ruzbeh \\ Hadavandi, Luca D'agostino
}

\section{- To cite this version:}

Dario Valentini, Giovanni Pace, Angelo Pasini, Lucio Torre, Ruzbeh Hadavandi, et al.. Fluid-Induced Rotordynamic Forces on a Whirling Centrifugal Pump. 16th International Symposium on Transport Phenomena and Dynamics of Rotating Machinery, Apr 2016, Honolulu, United States. hal-01890061

\section{HAL Id: hal-01890061 \\ https://hal.science/hal-01890061}

Submitted on 8 Oct 2018

HAL is a multi-disciplinary open access archive for the deposit and dissemination of scientific research documents, whether they are published or not. The documents may come from teaching and research institutions in France or abroad, or from public or private research centers.
L'archive ouverte pluridisciplinaire HAL, est destinée au dépôt et à la diffusion de documents scientifiques de niveau recherche, publiés ou non, émanant des établissements d'enseignement et de recherche français ou étrangers, des laboratoires publics ou privés. 


\title{
Fluid-Induced Rotordynamic Forces on a Whirling Centrifugal Pump
}

\author{
Dario Valentini ${ }^{1 *}$, Giovanni Pace ${ }^{1}$, Angelo Pasini $^{1}$, Lucio Torre ${ }^{2}$, Ruzbeh Hadavandi ${ }^{1}$, Luca d'Agostino ${ }^{3}$
}

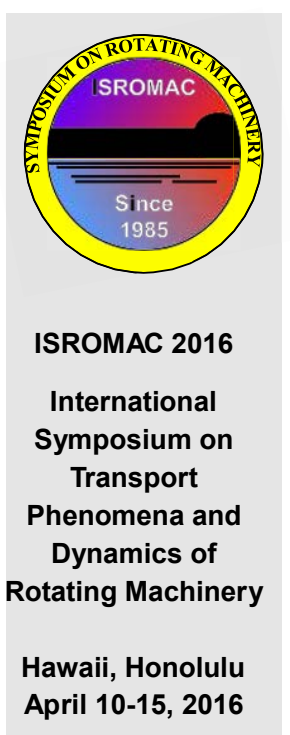

\begin{abstract} forces at negative whirl ratio.

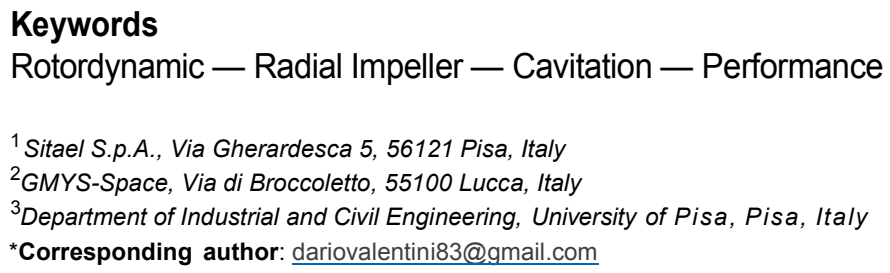

The experimental characterization of the rotordynamic fluid forces acting on a whirling centrifugal impeller have been investigated at different flow rates and cavitating conditions. The recently developed method for continuously measuring the rotordynamic forces at variable whirl ratios has been readapted and successfully applied for measuring the same forces at constant whirl ratio but variable cavitation number. The flowrate has a major influence on the stability of the rotordynamic forces at positive whirl ratios where a threshold flowrate separates the stable zone from the unstable. At negative whirl ratios, the normal force is typically unstable independently on the flow rate. Cavitation has always a destabilizing effect at positive whirl ratio while it can stabilize the rotordynamic

\section{INTRODUCTION}

Chemical rocket propulsion and its derivative concepts will continue to play a central role in future STSs (Space Transportation Systems), being the only viable technology capable of generating the relatively high levels of thrusts necessary for launch and most primary propulsion purposes in a large number of space missions.

Propellant feed turbopumps are an essential component of all primary propulsion concepts powered by Liquid Propellant Rocket Engines (LPREs). Rotordynamic forces, together with the flow instabilities possibly triggered by the occurrence of cavitation, are one of the universally recognized and most dangerous sources of vibrations in turbomachines $[1, \underline{2}, \underline{3}, 4]$. These forces can affect the impeller itself, and all the components of the machine [5]. The rotordynamic configuration of the Cavitating Pump Rotordynamic Test Facility (CPRTF, see [6] for further information) at SITAEL (formerly ALTA) is specifically intended for the analysis of steady and unsteady fluid forces, and moments acting on the impeller as a consequence of its whirl motion under cavitating or fully-wetted flow conditions, with special emphasis on the onset and development of lateral rotordynamic instabilities. Even if steady and rotordynamic forces acting on centrifugal pump impellers have already been extensively studied (see for example $[\underline{7}, \underline{8}]$ ), the influence of cavitation on rotordynamic fluid forces has not yet been investigated in great detail. Available experimental data mainly come from the work carried out at the California Institute of Technology $[1, \underline{2}, \underline{3}, \underline{9}, \underline{10}]$, and later at ALTA by the Chemical Propulsion Team $[11, \underline{12}, \underline{13}, \underline{14}]$.
Recently, an experimental campaign has been carried out at ALTA under ESA funding with the purpose of investigating the influence of the operational conditions (flow rate, suction pressure) and liquid temperature (inertial/thermal cavitation) on the rotordynamic forces acting on a six-bladed centrifugal impeller. In the experiments the impeller is subject to a whirl motion of given constant eccentricity and angular velocity. A special procedure, recently developed and validated at ALTA [13], has been used to measure the continuous spectrum of rotordynamic forces as functions of the whirl-to-rotational speed ratio for the first time on a centrifugal impeller. This procedure proved to be very effective in providing accurate and frequency-resolved information on the dependence of rotordynamic impeller forces on the whirl ratio and the operational conditions of the machine. In particular, the maxima and minima of such forces can be clearly identified, together with the general trend of their spectral behavior. Moreover the new procedure easily allows for the simultaneous evaluation of the rotordynamic forces along with the non-cavitating and suction performance of the test machine.

\section{EXPERIMENTAL APPARATUS}

The experimental activity reported in the present paper has been carried out in SITAEL's Cavitating illustrated in Fig. 1 and specifically designed for characterizing the performance of cavitating and/or non-cavitating turbopumps in a wide variety of alternative configurations: axial, radial or mixed flow, with or without an inducer [6]. The facility uses water as working fluid at temperatures up to $90{ }^{\circ} \mathrm{C}$ and is Pump Rotordynamic Test Facility (CPRTF), 
intended as a flexible apparatus readily adaptable to conduct experimental investigations on virtually any kind of fluid dynamic phenomena relevant to high performance turbopumps [6]. The pump housing and the inlet section can be easily adapted to host fullscale machines and inducers with different sizes and geometries used in space applications.

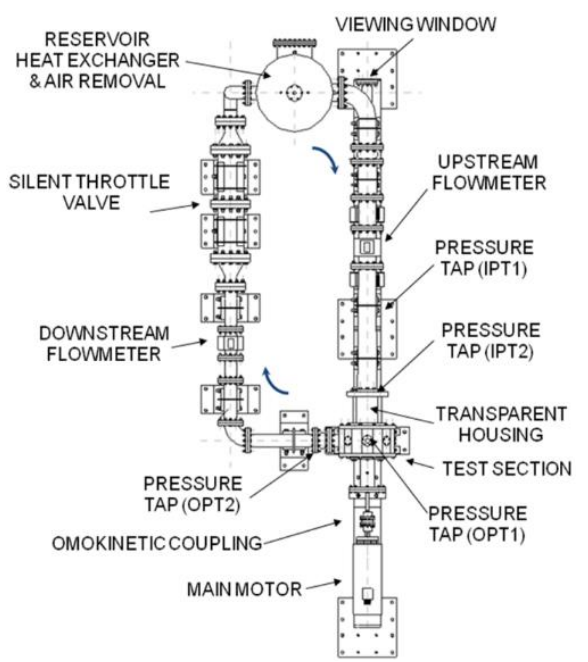

Fig. 1: The CPRTF lay-out in SITAEL S.p.A.

In its rotordynamic configuration, the facility instrumentation (transducers, optical devices, etc.) allows for the measure of:

1. The inducer inlet static pressure $p_{1}$ (cavitating regime, $\left.\sigma=\left(p_{1}-p_{v}\right) /\left(0.5 \rho \Omega^{2} r_{2}^{2}\right)\right)$

2. The pump total pressure rise $\Delta p_{t}$ (head coefficient, $\left.\Psi=\Delta p_{t} /\left(\rho \Omega^{2} r_{2}^{2}\right)\right)$

3. The volumetric flow rate on the discharge line (flow coefficient, $\Phi=\dot{V} /\left(\Omega r_{2}^{3}\right)$ )

4. The fluid temperature inside the main tank

5. The absolute angular position of the driving shaft

6. The absolute angular position of the eccentric shaft which generates the whirl motion

7. The forces acting on the impeller

The eccentricity generation is realized by means of a two-shafts mechanism. The shafts are assembled one inside the other by means of a double eccentric mount and their eccentricity can be finely adjusted from 0 to $2 \mathrm{~mm}$ before each test by changing the relative angular position of the double eccentric mount. The whirl motion is generated by a brushless motor driving the external shaft, while the impeller rotation is imparted by connecting the internal shaft to the main motor by means of an isokinetic coupling.

The forces and moments acting on the impeller are measured by means of a squirrel-cage rotating dynamometer connecting the shaft to the impeller in the test section. The rotating dynamometer, manufactured in one piece of phase hardening steel AISI $630 \mathrm{H} 1025$, consists of two flanges connected by four square cross-section posts acting as flexible elements. The deformation of the posts is measured by 40 semiconductor strain gauges arranged in 10 full Wheatstone bridges, which provide redundant measurements of the forces and moments acting on the impeller. Each bridge is temperature selfcompensated, with separate bipolar excitation and read-out for better reduction of cross-talking. The sizing of the sensing posts is the result of a trade-off between sensibility and structural resistance, operational stability and position control (stiffness). The current design of the dynamometer is optimized for a suspended mass of $4 \mathrm{~kg}$ with $70 \mathrm{~mm}$ gyration radius, an added mass of about $2 \mathrm{~kg}$ (based on the expected magnitude of the rotordynamic forces), a rotational speed of $3000 \mathrm{rpm}$ without eccentricity, and maximum rotational and whirl speeds up to 2000 rpm with $2 \mathrm{~mm}$ shaft eccentricity. The measurement errors of the dynamometer in the rotating frame $(z$ is the rotating axis direction) are reported in Table 1.

Table 1 Maximum measurement errors given by the rotating dynamometer.

\begin{tabular}{c|c} 
Component & Maximum Error \\
\hline \hline$F_{x}$ & $\pm 1.4 \mathrm{~N}$ \\
\hline$F_{y}$ & $\pm 1.1 \mathrm{~N}$ \\
\hline$F_{z}$ & $\pm 4.2 \mathrm{~N}$
\end{tabular}

The rotating dynamometer is placed between the impeller and the driving shaft, therefore the impeller is suspended. In order to reduce cantilever effects on the impeller shaft, the impeller has been recessed with respect to the optical access at the test section inlet. In this configuration, impeller blades are contained within the "inlet duct". A nominal clearance of $2 \mathrm{~mm}$ has been selected in order to accommodate sufficiently large whirl eccentricities for generating measurable rotordynamic forces without excessively increasing tip leakage effects.

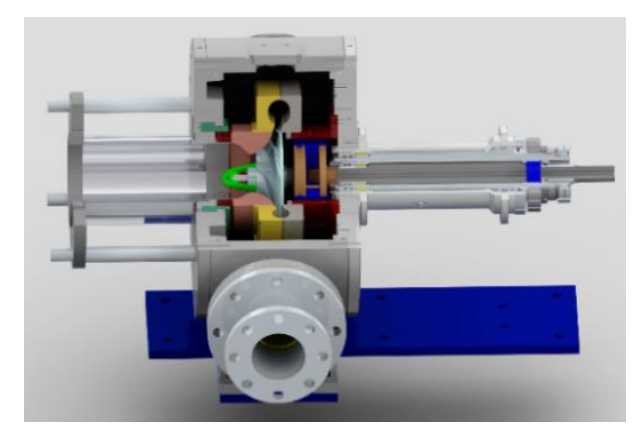

Fig. 2: Cut-out drawing of the Cavitating Pump Rotordynamic Test Facility (CPRTF).

\section{Test Article}

The VAMPIRE pump, manufactured in 7075-T6 aluminum alloy, comprises a six-bladed unshrouded impeller (Fig. 3), a vaneless diffuser and a singlespiral volute. At design conditions its predicted head coefficient and hydraulic efficiency are respectively 0.31 and $83 \%$. The test pump, whose main geometrical and operational data are reported in Table 2, has been designed by means of the reduced order model recently developed at Alta and described in $[15,16,17]$. Suitable settings for the 
inputs of the model allow for designing an inducer with dimensions fully compatible with the CPRTF configuration.

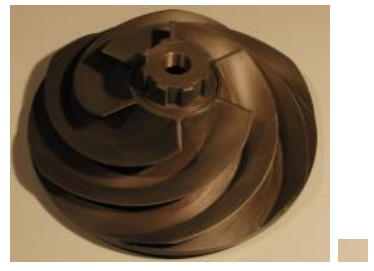

Fig. 3: The VAMPIRE impeller.

Table 2: Geometrical and operational design parameters of the VAMPIRE pump.

\begin{tabular}{|l|c|c|c|}
\hline Design flow coefficient & {$[--]$} & $\Phi$ & 0.092 \\
\hline Number of blades & {$[--]$} & $N$ & 6 \\
\hline Impeller outlet radius & $\mathrm{mm}$ & $r_{2}$ & 105 \\
\hline Axial length (fully-developed blade) & $\mathrm{mm}$ & $c_{a}$ & 46.4 \\
\hline Rotational Speed & {$[\mathrm{rpm}]$} & $\Omega$ & 1500 \\
\hline Design volumetric flowrate & {$[\mathrm{l} / \mathrm{s}]$} & $\dot{V}_{D}$ & 16.8 \\
\hline \hline Tip solidity & {$[--]$} & $\sigma_{T}$ & 2.26 \\
\hline Incidence tip angle @ design & $d e g$ & $\alpha$ & 17.4 \\
\hline Exit blade height & $\mathrm{mm}$ & $b_{2}$ & 10.5 \\
\hline Predicted design head coefficient & {$[--]$} & $\Psi_{D}$ & 0.31 \\
\hline Predicted specific velocity & {$[--]$} & $\Omega_{S}$ & 0.74 \\
\hline Predicted efficiency & $\%$ & $\eta$ & 83 \\
\hline
\end{tabular}

\section{EXPERIMENTAL PROCEDURE}

A circular whirl orbit has been imposed to the VAMPIRE impeller in order to determine the corresponding fluid-induced rotordynamic forces.

The generic components of the instantaneous forces acting on a whirling inducer are schematically shown in Fig. 4. The instantaneous force vector $\vec{F}$ can be expressed as the sum of a steady force $\overrightarrow{F_{0}}$ (not depending on the rotor eccentricity) and an unsteady force $\overrightarrow{F_{R}}$ generated by the eccentricity vector $\vec{\varepsilon}$ and represented by means of the rotordynamic matrix $\boldsymbol{A}$ which is generally function of the whirl speed:

$$
F=\vec{F}_{0}+\vec{F}_{R}=\vec{F}_{0}+[A] \vec{\varepsilon}
$$

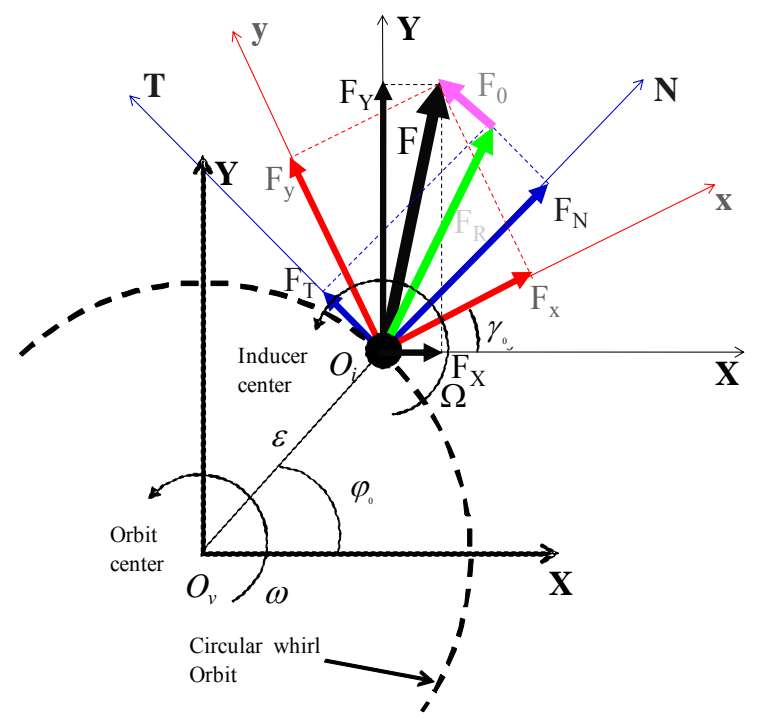

Fig. 4: Schematic representation of the rotordynamic forces in the laboratory and rotating reference frames.

It is convenient to express the rotordynamic force in terms of normal $\left(F_{N}\right)$ and tangential $\left(F_{T}\right)$ forces with respect to the whirl orbit:

$$
\begin{gathered}
F_{N}=\vec{F}_{R} \cdot \frac{\vec{\varepsilon}}{|\vec{\varepsilon}|} \\
F_{T}=\vec{F}_{R} \cdot \frac{\vec{\Omega} \wedge \vec{\varepsilon}}{|\vec{\Omega} \wedge \vec{\varepsilon}|}
\end{gathered}
$$

Therefore, the normal force $F_{N}$ is assumed positive when in outward direction, while $F_{T}$ is positive if it has the same direction of the impeller rotational speed $\Omega$ (Fig. 5).

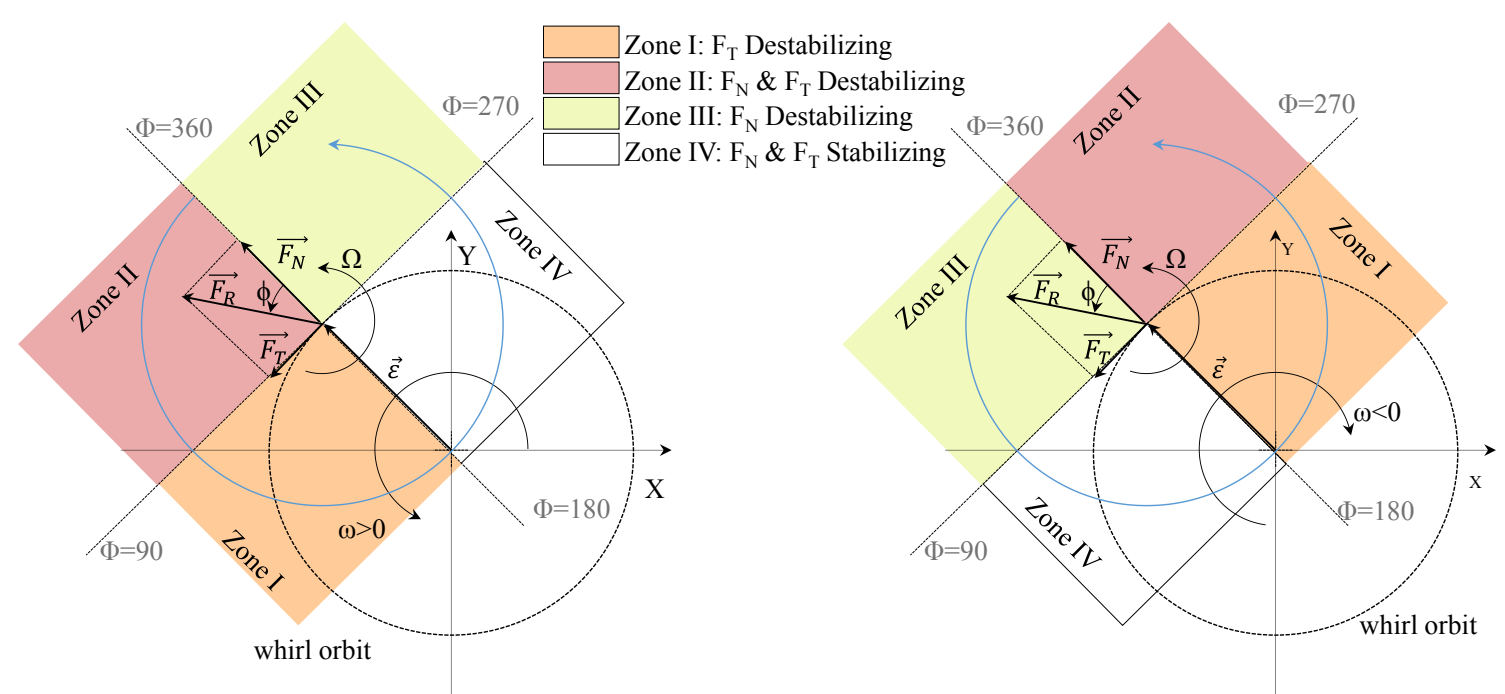

Fig. 5: Schematic of the stability regions of the rotordynamic force for positive (left) and negative (right) whirl ratios 
It is worth noting that the data presented in this paper only refer to fluid forces induced by rotor eccentricity. The effects of gravity, buoyancy and centrifugal force generated by the whirl motion on the rotor mass, as well as the steady fluid force on the impeller (like those induced by azimuthal asymmetries of the flow), have been subtracted from the total force read by the dynamometer.

The rotordynamic forces have been normalized as follows:

$$
F_{N}^{*}=\frac{F_{N}}{\pi \rho c_{a} \varepsilon \Omega^{2} r_{2}^{2}} \quad F_{T}^{*}=\frac{F_{T}}{\pi \rho c_{a} \varepsilon \Omega^{2} r_{2}^{2}}
$$

where $\rho$ is the fluid density, $c_{a}$ is the axial length of the impeller blades (axial chord), $\varepsilon$ is the radius of the whirl orbit (eccentricity), $\Omega$ is the inducer rotational speed and $r_{2}$ is the impeller outlet tip radius.

Experiments have been carried out following both the classical discrete approach [18] and a continuous one (named chirp) illustrated in detail in a previous paper [13]. This method allows for obtaining a continuous spectrum of the rotordynamic force as a function of the whirl ratio from the data acquired in a single experiment characterized by an imposed continuous variation of the whirl speed. Moreover, it overcomes most of the frequency resolution limitations connected with the traditional approach, which is based on experiments at fixed values of the whirl speed $[1, \underline{3}, 10]$.

For effective rejection of the measurement noise in discrete tests the duration of each run must be a large multiple of the fundamental reference period, which in turn is simultaneously an integer multiple of both the whirl and rotational periods of the impeller. On the other hand, in continuous tests the whirl speed is varied at a slow but constant rate, while the rotordynamic forces are evaluated by averaging the measurements over a suitable time-window where the whirl speed can be assumed to remain nearly constant. Time shift of the averaging window then provides a virtually continuous spectrum of the rotordynamic force as a function of the whirl frequency. In this procedure the errors associated with the non-periodic initial/final positions of the rotating shafts and with the slow drift of the whirl frequency ratio during the acquisition time window are therefore neglected. Comparison with the results obtained using the discrete approach fully confirmed the validity of this assumption [19,12], greatly simplifying the design and execution of the experiments.

\section{Test Matrix}

A first experimental campaign has been carried out on the VAMPIRE pump aimed at understanding how the rotordynamic forces are influenced by:

- Flowrate (flow coefficient, $\Phi$ )

- Cavitation regime (cavitation number, $\sigma$ )

All tests have been carried out with whirl eccentricity $\varepsilon=1.130 \pm 0.05 \mathrm{~mm}$ and impeller rotational speed, $\Omega=1750 \mathrm{rpm}$, which represent a suitable compromise between generation of measurable rotordynamic forces and structural integrity of the rotating dynamometer under the resulting centrifugal forces on the rotor. Only subsynchronous whirl ratios have been investigated, with both positive and negative values up to \pm 0.7 $(\omega / \Omega= \pm 0.1, \pm 0.3, \pm 0.5 \pm 0.7)$ for the discrete approach). Nine different combinations of flow rate and cavitating conditions have been considered, as summarized in the test matrix of Table 3.

The flow rate effect on the rotordynamic forces has been studied at three flow coefficients $(\Phi=$ $0.074, \Phi=0.092$, and $\Phi=0.110$ ), corresponding respectively to $80 \%, 100 \%$, and $120 \%$ of the design flow coefficient. Three (nominal) values of the cavitation number $\sigma_{N}$ have been selected in order to obtain different cavitating conditions: non-cavitating (NC, $\sigma_{N}=0.6$ ), slightly cavitating (SC, $\sigma_{N}=0.11$ ) and highly cavitating $\left(\mathrm{HC}, \sigma_{N}=0.08\right)$. All tests have been carried out in water at room temperature $\left(T \cong 20^{\circ} \mathrm{C}\right)$.

Table 3 Test matrix.

\begin{tabular}{|c|c|}
\hline$\Phi$ & $\sigma_{N}$ \\
\hline $\begin{array}{c}0.074|0.092| 0.110 \\
\left(0.8 \Phi_{D}\left|\Phi_{D}\right| 1.2 \Phi_{D}\right)\end{array}$ & $\begin{array}{l}0.6|0.11| 0.08 \\
(\mathrm{NC}|\mathrm{SC}| \mathrm{HC})\end{array}$ \\
\hline
\end{tabular}

After the first campaign, comparative analysis of the continuous spectra of the rotordynamic forces clearly indicated the occurrence of general trends and suggested the presence of particular operating points, hereafter indicated as invariance-points since they appear to be unaffected by changes of specific operational parameters. In the following, a second campaign is reported based on these observations. In particular, two different kinds of tests have been conducted:

- Non-cavitating pumping performance characterization under forced whirl motion

- Suction performance characterization under forced whirl motion

Specifically, these experiments consist in the usual characterization of the hydraulic performance of the machine under cavitating/non-cavitating conditions (see for example [6,20]) in conjunction with the simultaneous measurement of the rotordynamic fluid forces on the impeller under the imposed whirl motion for fixed values of $\omega / \Omega$. The data reduction is based on the recent chirp approach [13] suitably readapted for the first time during this experimental campaign.

\section{RESULTS AND DISCUSSION}

In the following, the rotordynamic forces are first reported both in terms of $F_{N}^{*}, F_{T}^{*}$, their nondimensional modulus $\left|\overrightarrow{F_{R}^{*}}\right|=\sqrt{\left(F_{N}^{*}\right)^{2}+\left(F_{T}^{*}\right)^{2}}$, and phase angle $\phi$ as functions of the whirl ratio $\omega / \Omega$. The phase angle addresses the direction of the rotordynamic force. The phase angle starts from the 
whirl outward eccentricity direction and is positive according to the main rotational speed (Fig. 5). Recalling that these forces are destabilizing when they tend to increase the whirl eccentricity or sustain its rotation, Fig. 5 shows areas of the stability regions for positive and negative whirl ratios with regard to the phase angle. Only relevant results obtained from the experimental campaign described in Table 3 are here reported and discussed. The results of discrete and continuous tests are respectively indicated by individual points and lines, using equal colors when referring to the same operating conditions. The excellent agreement of the two sets of measurements effectively confirms the validity of the continuous test procedure. In general the rotordynamic is oriented towards zone III (Fig. 5) for negative whirl ratios and starts rotating towards zone I with different $\omega / \Omega$ cross-values depending on the specific operational conditions as will be described in the following.

\section{Flowrate Effects}

As represented in Fig. 6, the tangential component is stabilizing and almost not affected by the flowrate for negative whirl ratios whereas for positive whirl ratios a greater value of the flow coefficient corresponds to a greater destabilizing intensity of $F_{T}^{*}$. On the other hand, the normal component $\left(F_{N}^{*}\right)$ is strongly affected by the flow coefficient variation. In general, all the curves pass
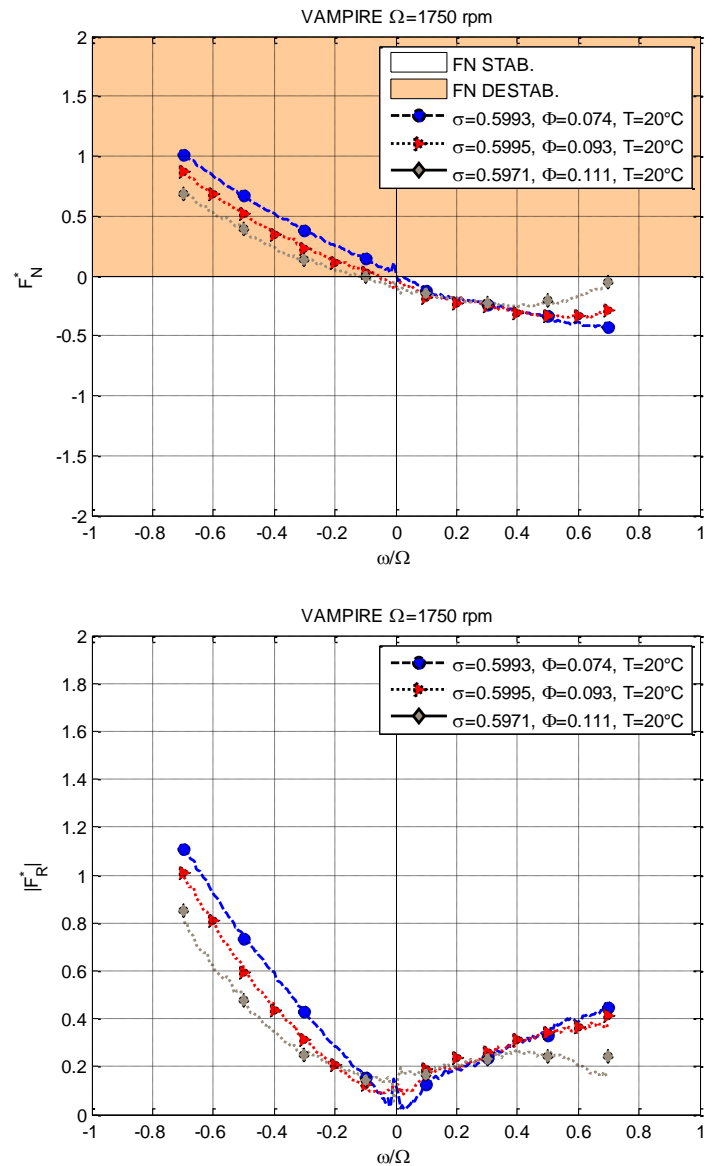

for the same value of $F_{N}^{*}$ for $\omega / \Omega \cong 0.2$ and then "rotate" around this fixed point maintaining qualitatively the same parabolic behavior. Therefore, they show variable threshold values of the whirl ratio from the unstable to the stable region and vice versa. The rotordynamic force module $\left(\left|F_{R}^{*}\right|\right)$ changes consequently in terms of both shape and magnitude. In general, the lower the flow coefficient the greater the intensity of the rotordynamic force. From the analysis of the phase $(\phi)$ it is evident that only the condition with the minimum flowrate under non cavitation condition $(\Phi=0.111, \sigma \cong 0.6)$ pass through the fully stable region for a reasonable range of whirl ratios $(0<\omega / \Omega \leq 0.5)$. Table 4 summarizes some of the detected trends exploited for selecting the whirl ratios for further experimental investigation.

Table 4: Relevant detected behaviors of the rotordynamic forces for increasing flowrates (increasing $\Phi)$.

\begin{tabular}{|c|c|}
\hline Relevant Detected Behavior & $\boldsymbol{\omega} / \mathbf{\Omega}$ \\
\hline \hline module reduction of $F_{R}$ and $F_{N}$ & -0.5 \\
\hline $\begin{array}{c}F_{N}=\text { constant }\left(F_{N} \cong-0.2\right) \text { and } \\
\text { stable to unstable transition of } F_{T}\end{array}$ & 0.2 \\
\hline module reduction of $F_{R}$ and $F_{N}$ & 0.7 \\
\hline
\end{tabular}
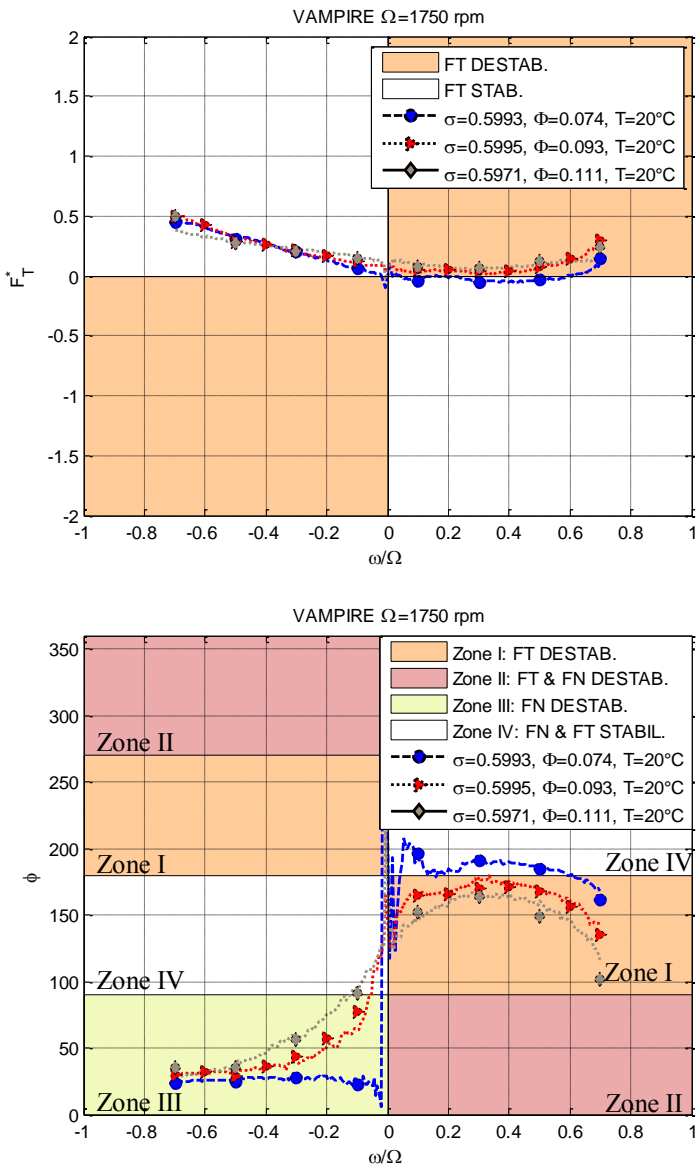

Fig. 6: Continuous and discrete tests: effect of the flow coefficient $\Phi$ on $F_{N}^{*}, F_{T}^{*},\left|F_{R}^{*}\right|$, and $\phi$ under non-cavitating regime. 

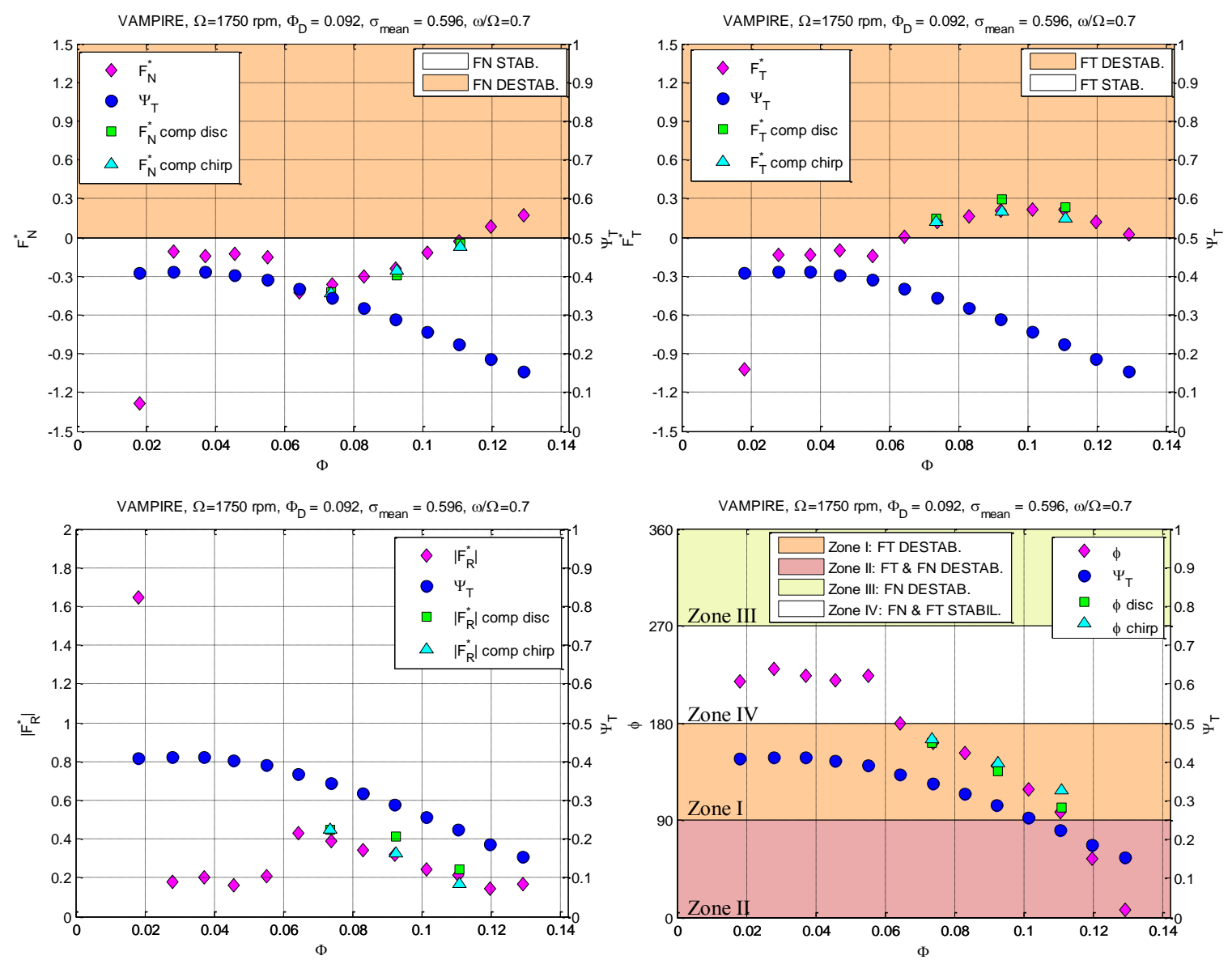

Fig. 7: $F_{N}^{*}, F_{T}^{*},\left|F_{R}^{*}\right|$, and $\phi$ together with the non-cavitating performance for $\omega / \Omega=0.7$.

Fig. 7 to Fig. 9 illustrate the behavior of the rotordynamic fluid force on the test pump as a function of the flowrate under non-cavitating conditions for fixed values and the whirl ratio $\omega / \Omega$ as specified in Table 4.

1. the rotordynamic force relevant parameter, indicated by purple diamonds, with values to be read on the left $y$-axis

2. the total head coefficient $\left(\Psi_{T}\right)$ of the pump, indicated by blue circles, with values to be read on the right $y$-axis

Where available, each figure also reports the results obtained during the previous experimental campaign for comparison. They are indicated by green squares when referring to the discrete tests and by cyan triangles in the case of continuous (chirp) tests.

The same trends and invariance points reported in Table 4 have also been observed in the present campaign. However, it is worth noting that these trends are sometimes limited only to the flowrates tested during the previous campaign and cannot be extended to other ranges of the operational flowrates of the machine.

As underlined in [21], the flowrate determines the hydrodynamic load on the impeller blades of the machine and is therefore one of the most important parameters to be taken into consideration for the analysis of fluid-induced rotordynamic forces. If compared to an axial machine (see [21]), for instance, the fluid induced rotordynamic force is very regular for the VAMPIRE pump, even though the flowrate has a more irregular and unpredictable impact on the rotordynamic force behavior for positive whirl ratios (Fig. 7 and Fig. 8) than for negative values (Fig. 9). This asymmetry is most likely connected with a coupling between the whirl motion and the backflow structure that can occur. The complex flow structures connected with the reverse flow depends on the flowrate value and rotate around the machine with a velocity governed by the main rotational speed of the impeller. Nevertheless, for $\omega / \Omega=0.2$ (Fig. 8) the acting forces are quite low, therefore the absolute variations are of less importance. For highly negative whirl ratio values $(\omega / \Omega<0$, Fig. 9) where the backflowstructure and the whirl motion are counter-rotating, the trend of the rotordynamic force is quite regular. On the other hand, for positive values $(\omega / \Omega>0$, Fig. 7 and Fig. 8), when the whirl motion and the rotational verse of the backflow-structure are in the same direction, the irregularity of the fluid induced rotordynamic force increases. This effect suggests the presence of a coupling between the two phenomena that tends to be exciting when they rotate in the same direction. 

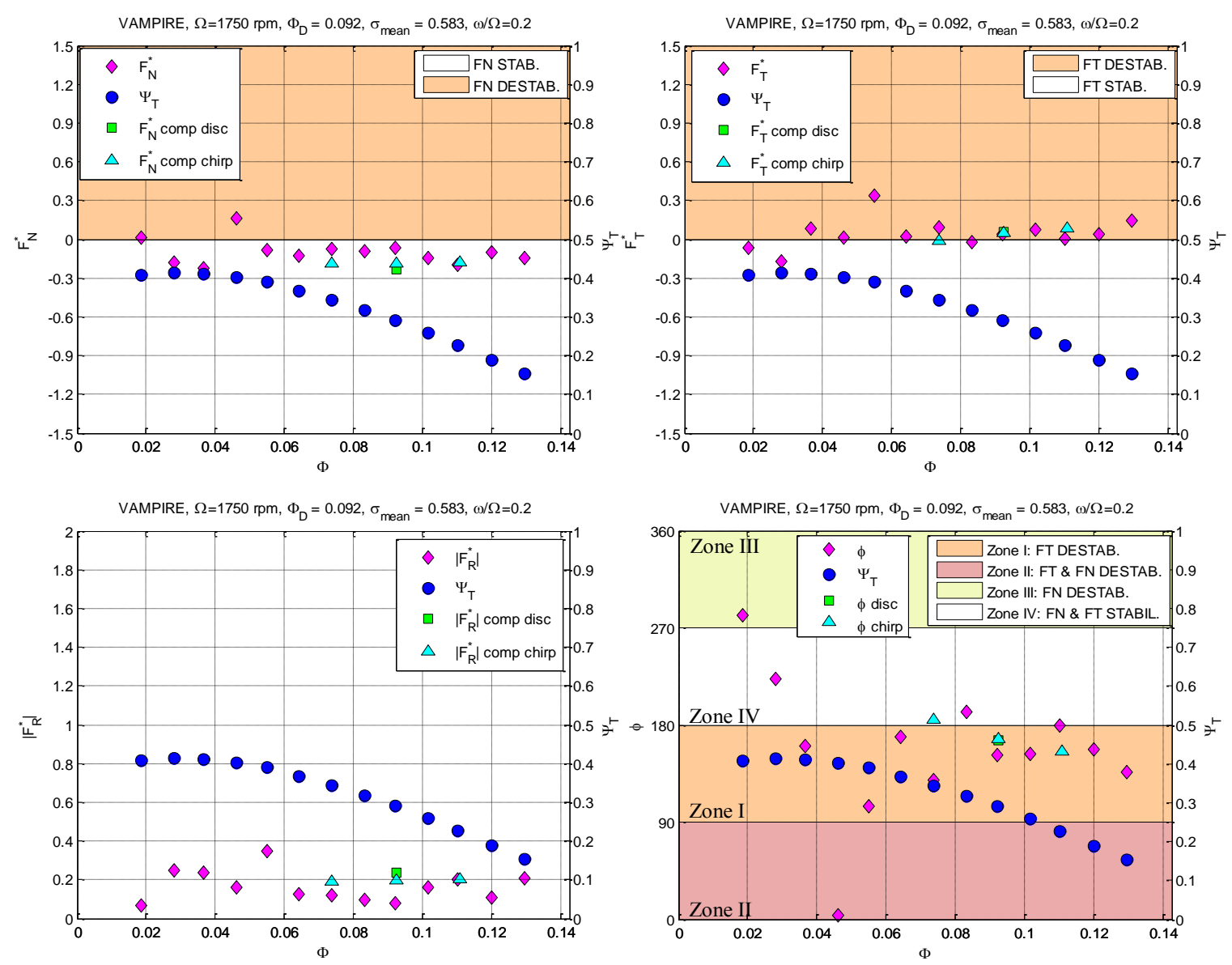

Fig. 8: $F_{N}^{*}, F_{T}^{*},\left|F_{R}^{*}\right|$, and $\phi$ together with the non-cavitating performance for $\omega / \Omega=0.2$.
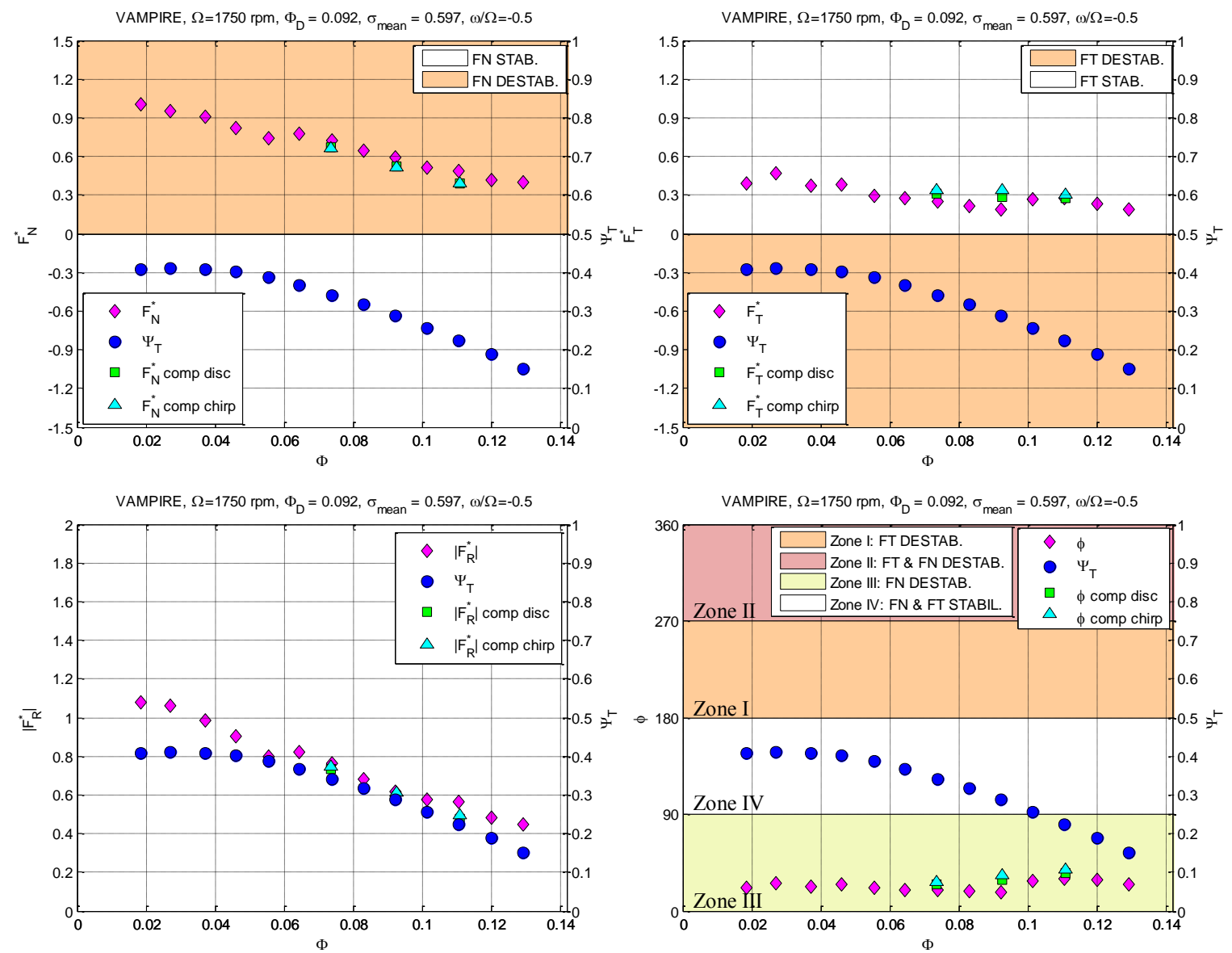

Fig. 9: $F_{N}^{*}, F_{T}^{*},\left|F_{R}^{*}\right|$, and $\phi$ together with the non-cavitating performance for $\omega / \Omega=-0.5$. 


\section{Cavitation Effects}

As shown in Fig. 10, the tangential component of the rotordynamic force is not strongly affected by the variation of the cavitation number $\sigma$. The analysis of the normal component points out a dependence on the cavitating condition for $\omega / \Omega>0.5$ for all the flow coefficients. Moreover, the highest investigated flow coefficient $(\Phi=0.111$, represented in Fig. 10) shows a dependence on the cavitation condition for all the whirl ratios. The module of the rotordynamic force is consequently not so strongly affected. In general, for negative whirl ratios the module is higher than the one for positive whirl ratios and it decreases with the absolute value of $\omega / \Omega$. The analysis of the phase suggests that the cavitation has generally a destabilizing effect for positive whirl ratios whereas it has a stabilizing effect for negative whirl ratios. The results have been used to select the whirl ratios as summarized in Table 5 for further investigation.

Fig. 11 to Fig. 13 illustrate the behavior of the rotordynamic fluid force on the test pump as a function of the development of cavitation for fixed values of the flowrate $\left(\Phi=1.2 \Phi_{\mathrm{D}}=0.111\right)$ and the whirl ratio $\omega / \Omega$ as specified in Table 5 .
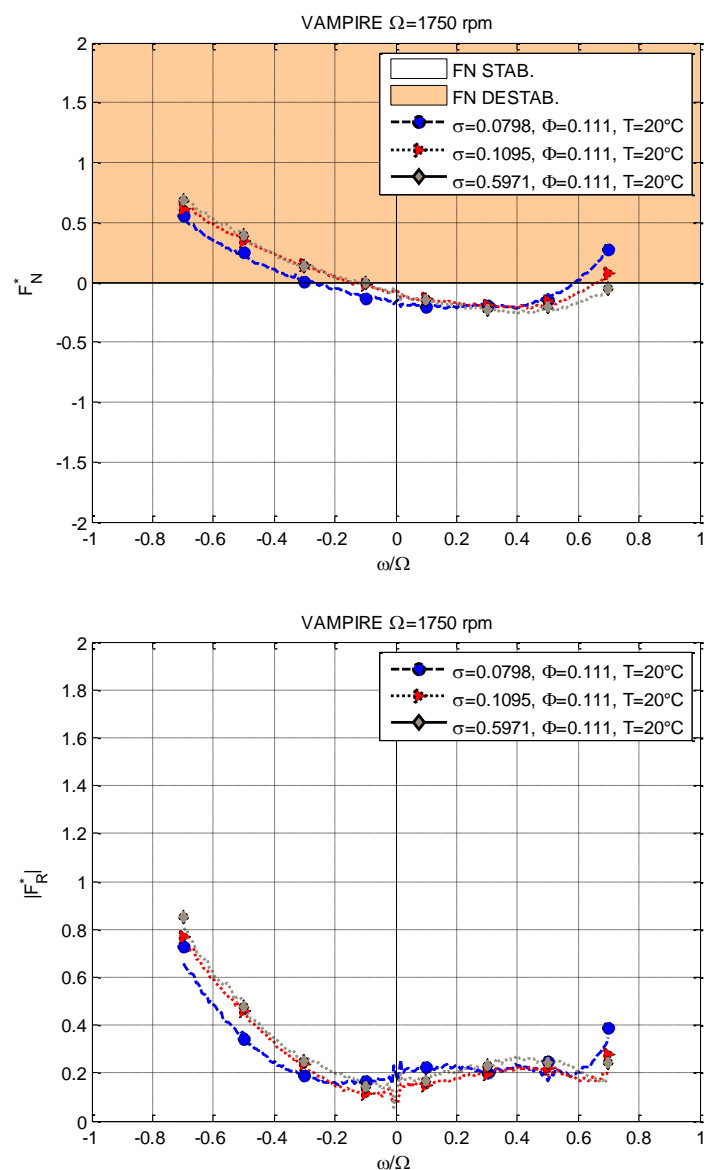

1. the rotordynamic force (in terms of either its normal and tangential components or its amplitude and phase), indicated by purple diamonds with values to be read on the left $y$ axis

2. the head coefficient normalized w.r.t. its noncavitating value, $\Psi / \Psi_{N C}$, indicated by blue circles with values to be read on the right $y$-axis

As for the non-cavitating case, the results obtained in the previous campaign are almost perfectly matched by present findings, thus confirming the capability of the continuous testing procedure to effectively provide reliable results. The summary reported in Table 5 is generally confirmed.

Table 5: Relevant detected behaviors of the rotordynamic forces for increasing level of cavitation (decreasing $\sigma$ ), $\Phi=0.111$.

\begin{tabular}{|c|c|}
\hline Relevant Detected Behavior & $\boldsymbol{\omega} / \mathbf{\Omega}$ \\
\hline \hline $\begin{array}{c}F_{T}=\text { constant }\left(F_{T} \cong 0.27\right) \text { and } \\
\text { module reduction of } F_{N} \text { and } F_{R}\end{array}$ & -0.5 \\
\hline Destabilizing effect on $F_{T}$ & 0.5 \\
\hline
\end{tabular}
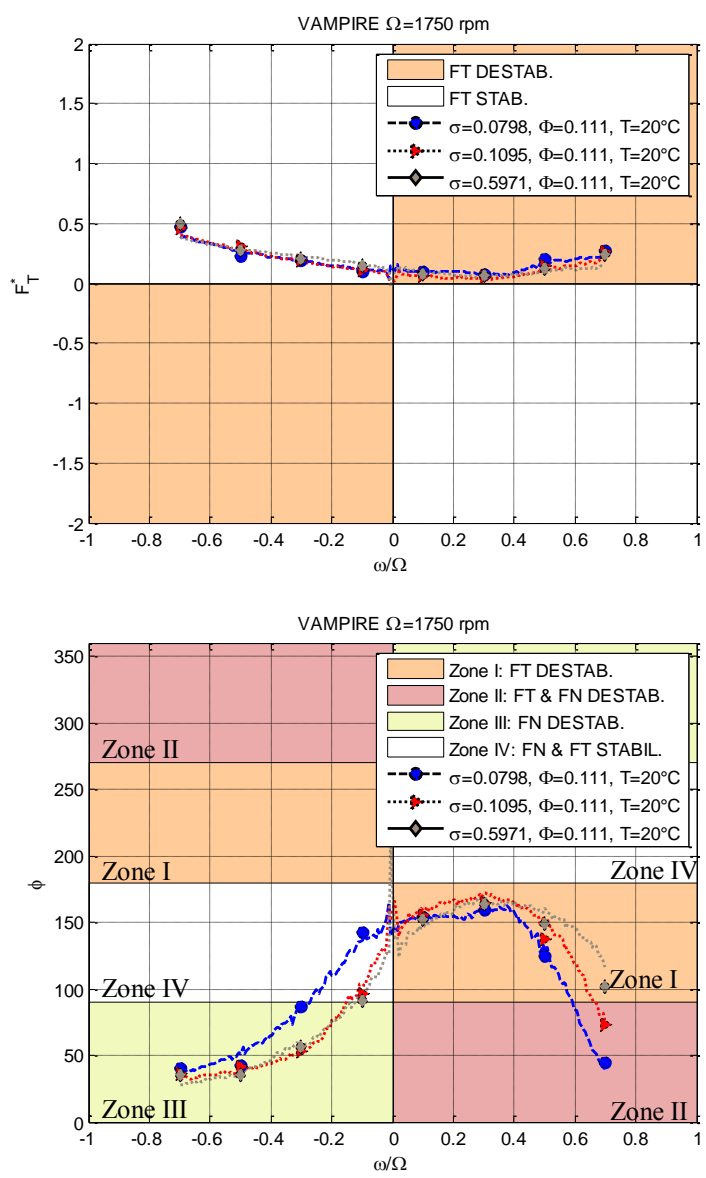

Fig. 10: Continuous and discrete tests: effect of different cavitating regimes $\sigma$ on $F_{N}^{*}, F_{T}^{*},\left|F_{R}^{*}\right|$, and $\phi$ for $\Phi=1.2 \Phi_{D}$. 

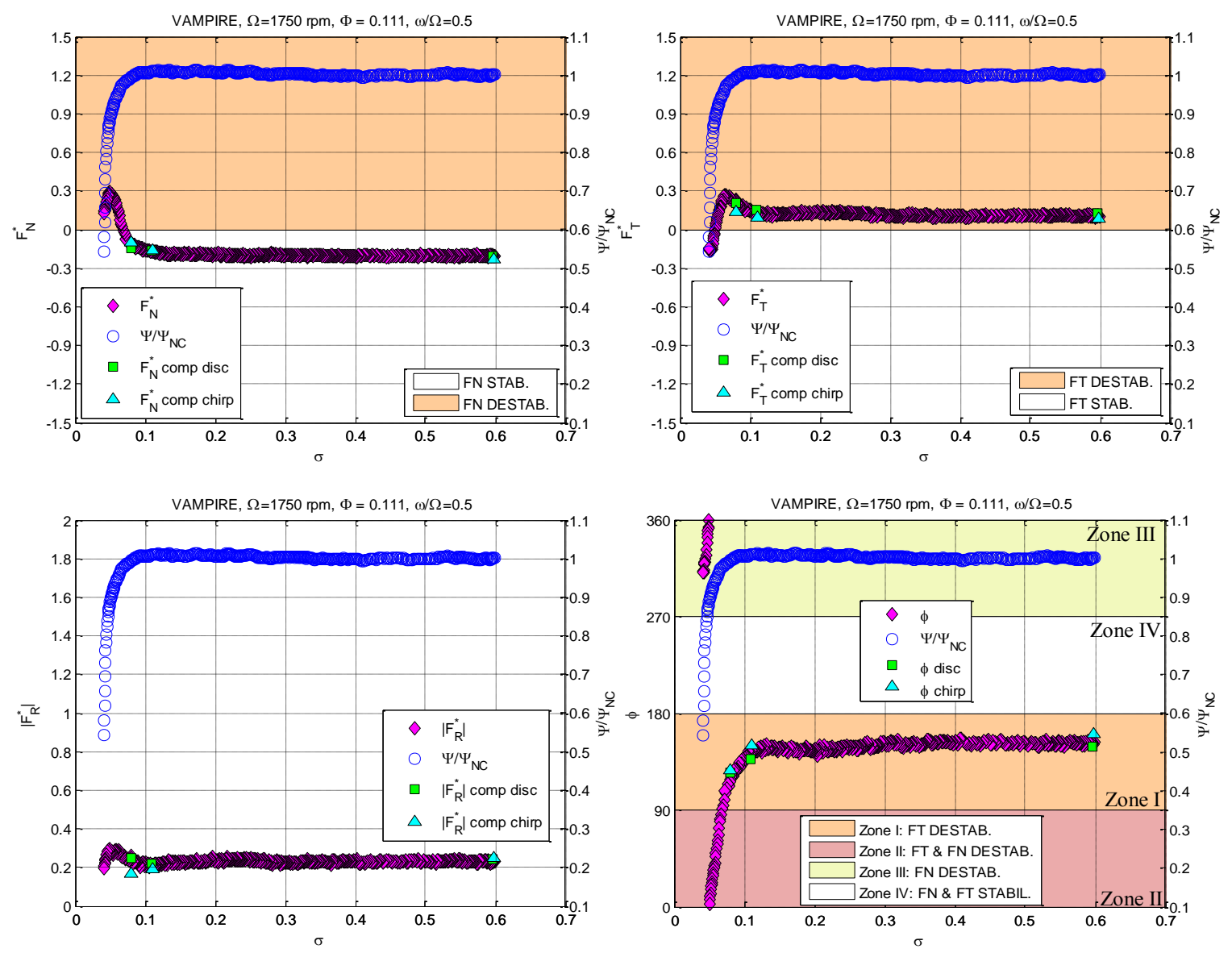

Fig. 11: $F_{N}^{*}, F_{T}^{*},\left|F_{R}^{*}\right|$, and $\phi$ together with the suction performance for $\omega / \Omega=0.5, \Phi=1.2 \Phi_{D}=0.111$.

The tests show how the normal and tangential components start to vary in correspondence of the beginning of the head degradation. This is most likely due to the correspondent change of the blade loading which, in turn, mainly affects the forces acting on the machine, and thus on the shaft.

Regarding the tested positive whirl frequency (Fig. 11), for increasing level of cavitation both the components move toward greater unstable conditions until they reach a maximum and then they suddenly drop. Consequently the rotordynamic force module shows the same trend with regard to the cavitating regime $(\sigma)$, even if, quantitatively, the module shows minor variations. On the other hand, an analysis of the phase demonstrates how the rotordynamic force practically rotates of $180^{\circ} \mathrm{C}$ from the beginning of the head degradation to the end of the test.

For the tested negative whirl ratio (Fig. 12 and Fig. 13), the normal component does not show the peak encountered in the positive whirl ratio case, it starts to drop down in correspondence of the beginning of the head degradation and continues to fall together with the head. On the other hand the tangential component shows a minimum for increasing level of cavitation just after the beginning of the head drop, while the module of the rotordynamic force is characterized by the same behavior of the tangential component.
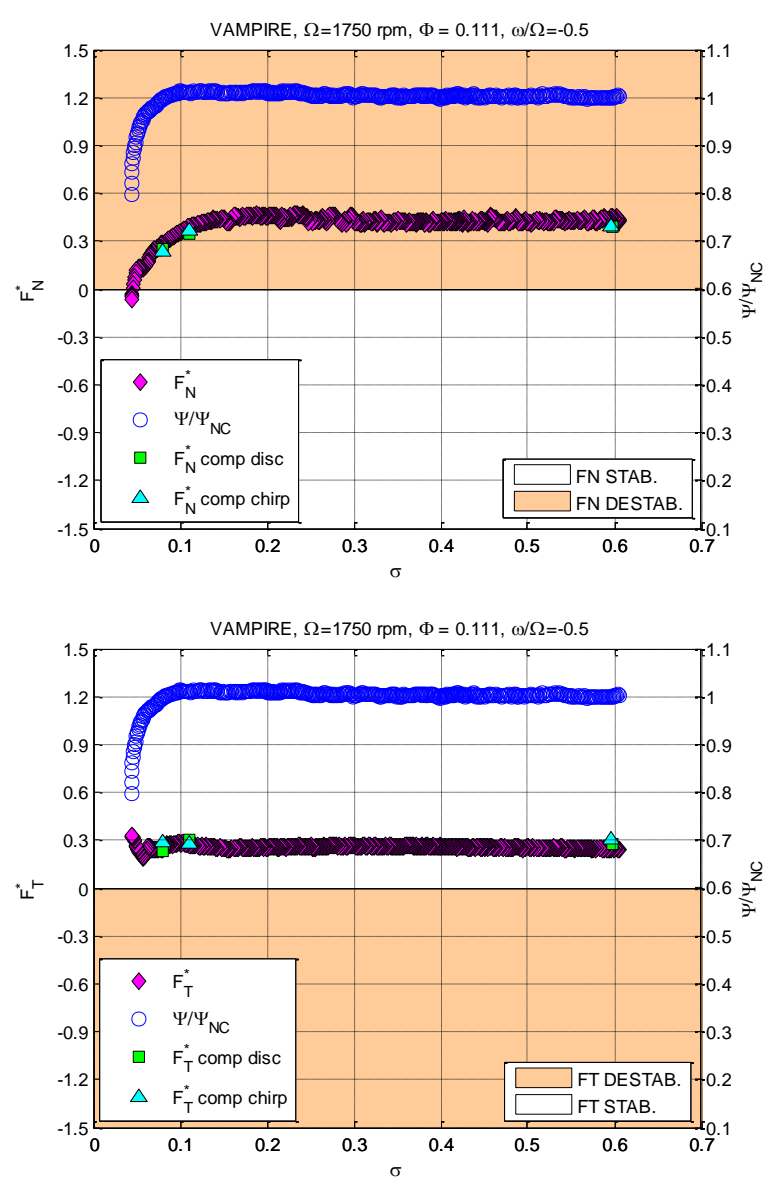

Fig. 12: $F_{N}^{*}$ and $F_{T}^{*}$ together with the suction performance for $\omega / \Omega=-0.5, \Phi=1.2 \Phi_{D}=0.111$. 

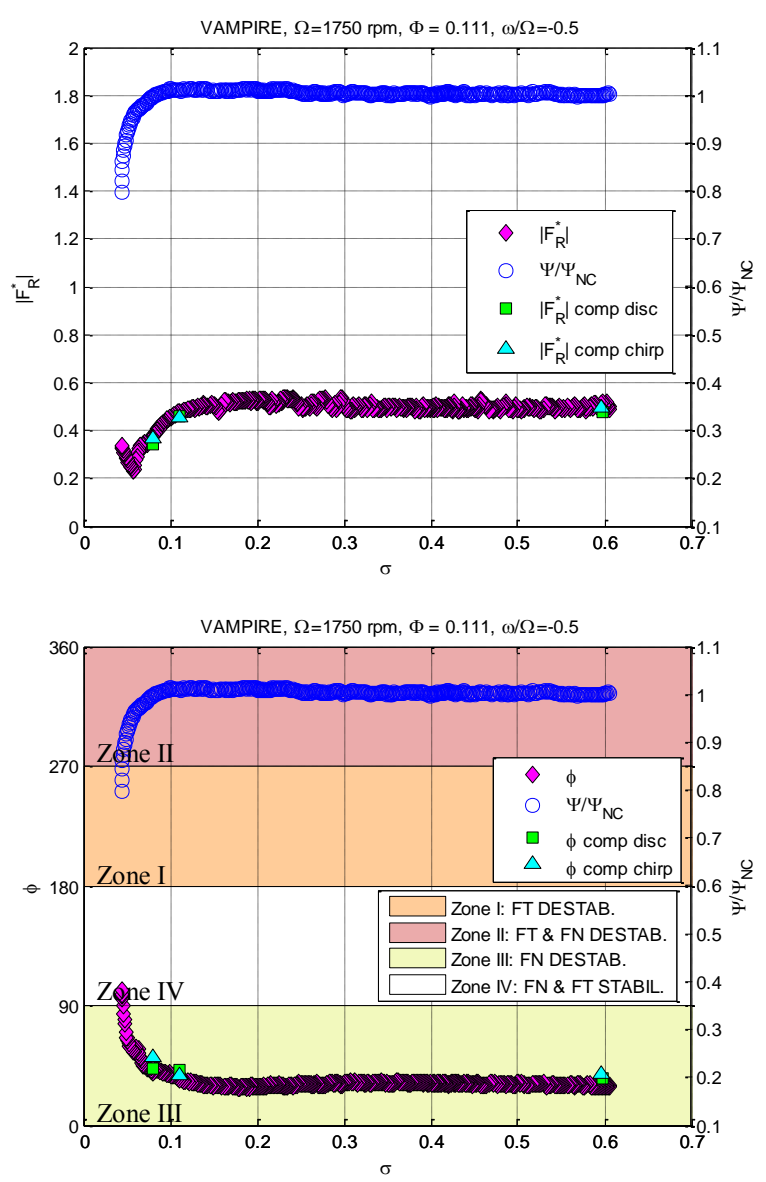

Fig. 13: $\left|F_{R}^{*}\right|$ and $\phi$ together with the suction performance for $\omega / \Omega=0.5, \Phi=1.2 \Phi_{D}=0.111$.

\section{CONCLUSION}

The rotordynamic forces acting on an unshrouded radial impeller have been measured at three different flow coefficients in sub-synchronous whirl ratio range $( \pm 0.7)$. Three relevant whirl ratios (such as $0.7,0.2$ and -0.5 ) have been selected for further experimental investigations aimed at assessing the influence of the flow coefficient on the rotordynamic forces at constant whirl ratio.

The main results can be summarized as follows:

- for highly positive whirl ratio (such as 0.7 ), there is a threshold value of the flow rate below which the overall rotordynamic force is stable and maintain the same modulus and direction. Beyond the threshold, the force becomes unstable and tends to decrease as the flow rate increases;

- for slightly positive whirl ratio (such as 0.2 ), the forces are of low magnitude but unstable. A general trend with the flow rate is not detectable;

- for negative whirl ratio (such as -0.5), the magnitude of the force decreases with the flow rate while its direction remains the same (unstable in the normal component).

Moreover, the influence of cavitation on the

rotordynamic forces at constant flow rate $(\Phi=$ $\left.1.2 \Phi_{\mathrm{D}}\right)$ has been assessed.

For positive whirl ratio $(\omega / \Omega=0.5)$, as the inlet pressure decreases, the rotordynamic force first increases up to a maximum and then slightly decreases to the same value in non-cavitating condition. In terms of direction, the force becomes more unstable with the increase of cavitation.

For negative whirl ratio $(\omega / \Omega=-0.5)$, the modulus of the rotordynamic force decreases with the reduction of the cavitation number. Moreover, the direction of the force moves toward the stable zone. Finally, at cavitation breakdown the force becomes fully stable.

$\boldsymbol{A}$

$c_{a}$

F

$p_{1}$

$p_{v}$

$r_{2}$

$T$

$\dot{V}$

$\Delta p_{t}$

$\alpha$

$\mathcal{E}$

$\phi$

$\Phi$

$\Psi$

$\Psi$

$\sigma$

$\sigma_{T}$

$\rho$

$\omega$

$\Omega$

\section{Subscript}

D design

$N \quad$ normal force, nominal

$T$ tangential force, total

$R \quad$ rotordynamic force

$x, y, z \quad$ relative frame

$X, Y, Z$ absolute frame

0 steady force 
Superscript

$q^{*} \quad$ normalized value of $\mathrm{q}$

$\vec{q} \quad$ vector of $\mathrm{q}$

Acronyms

NC non-cavitating

SC slightly cavitating

HC highly cavitating

\section{ACKNOWLEDGEMENTS}

The present work has been supported by the European Space Agency under Contract No. $4000102585 / 10 / \mathrm{NL} / \mathrm{Sfe}$. The authors would like to express their gratitude to Dr. Giorgio Saccoccia, ESA-ESTEC, Nordwijk, The Netherlands, for his constant attention and encouragement.

\section{REFERENCES}

${ }^{[1]}$ Bhattacharyya A., Acosta A.J., Brennen C.E., and Caughey T.K., 1997, "Rotordynamic Forces in Cavitating Inducers", J. Fluids Eng., 119(4), pp. 768774. DOI: $10.1115 / 1.2819496$

${ }^{[2]}$ Bhattacharyya A., 1994, "Internal Flows and Force Matrices in Axial Flow Inducers", Ph.D. Thesis, California Institute of Technology, Pasadena.

${ }^{[3]}$ Franz R.J., 1989, "Experimental investigation of the effect of cavitation on the rotordynamic forces on a whirling centrifugal pump impeller", Ph.D. Thesis, California Institute of Technology, Pasadena.

${ }^{[4]}$ Rosenmann W., 1965, "Experimental Investigations of Hydrodynamically Induced Shaft Forces With a Three Bladed Inducer," in ASME Symp. on Cavitation in Fluid Machinery, pp. 172-195.

${ }^{[b]}$ Ehrich F. and Childs S.D., 1984, "Self-Excited Vibrations in High Performance Turbomachinery", Mechanical Engineering, pp. 66-79.

${ }^{[6]}$ Pace G., Pasini A., Torre L., Valentini D., and d'Agostino L., 2012, "The Cavitating Pump Rotordynamic Test Facility at ALTA SpA: Upgraded Capabilities of a Unique Test Rig," in Space Propulsion Conference, Bordeaux, France.

${ }^{[7]}$ Hergt P. and Krieger P., 1969, "Paper 10: Radial Forces in Centrifugal Pumps with Guide Vanes," in Proceedings of the Institution of Mechanical Engineers, vol. 184, pp. 101-107.

${ }^{[8]}$ Suzuki T. et al., 2007, "Measurements of Rotordynamic Forces on an Artificial Heart Pump Impeller", J. Fluids Eng., 129(11), pp. 1422-1427. DOI: $10.1115 / 1.2786477$

${ }^{[9]}$ Brennen C.E., Franz R., and and Arndt N., 1988, "Effects of Cavitation on Rotordynamic Force
Matrices," in 3rd Earth to Orbit Propulsion Conf., vol. 3012, Huntsville, AL, USA, pp. 227-239.

${ }^{[10]}$ Brennen C.E., Franz R., and Arndt N., 1988, "Rotor/Stator Unsteady Pressure Interaction," in 3rd Earth to Orbit Propulsion Conf., vol. 3012, Huntsville, AL, USA, pp. 240-253.

[11] Torre L., Pasini A., Cervone A., and d'Agostino L., 2011, "Experimental Characterization of the Rotordynamic Forces on Space Rocket Axial Inducers", J. Fluids Eng., 133(10), p. 101102. DOI: 10.1115/1.4005100

[12] Torre L., Cervone A., Pasini A., and d'Agostino L., 2011, "Experimental Characterization of Thermal Cavitation Effects on Space Rocket Axial Inducers", J. Fluids Eng, 133(11), p. 111303. DOI:

10.1115/1.4005257

[13] Pasini A., Torre L., Cervone A., and d'Agostino L., 2011, "Continuous Spectrum of the Rotordynamic Forces on a Four Bladed Inducer", J. Fluids Eng, 133(12), p. 121101. DOI: 10.1115/1.4005258

${ }^{[14]}$ Valentini D., Pace G., Torre L., Pasini A., and d'Agostino L., 2014, "Rotordynamic Forces on a Three Bladed Inducer Under Forced Whirl Motion Operating at Different Conditions," in 15th International Symposium on Transport Phenomena and Dynamics of Rotating Machinery, Honolulu, Hawaii, USA.

[15] d'Agostino L., Pasini A., and Valentini D., 2011, "A Reduced Order Model for Preliminary Design and Performance Prediction of Radial Turbopumps," in 47th AIAA/ASME/SAE/ASEE Joint Propulsion Conference \& Exhibit, San Diego, California, USA.

[16] d'Agostino L. et al., 2012, "A REDUCED ORDER MODEL FOR OPTIMAL CENTRIFUGAL PUMP DESIGN," in 14th International Symposium on Transport Phenomena and Dynamics of Rotating Machinery, ISROMAC-14, Honolulu, HI, USA.

${ }^{[17]}$ Valentini D., Pasini A., Pace G., Torre L., and d'Agostino L., 2013, "Experimental Validation of a Reduced Order for Radial Turbopump Design," in 49th AIAA/ASME/SAE/ASEE Joint Propulsion Conference, San Jose, California, USA.

${ }^{[18]}$ Jery B., 1987, "Experimental Study of Unsteady Hydrodynamic Force Matrices on Whirling Centrifugal Pump Impellers", Report N. E200.22, Pasadena.

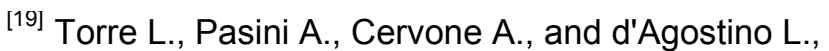
2011, "Continuous Spectrum of the Rotordynamic Forces on a Four Bladed Inducer," in ASME-JSMEKSME Joint Fluids Engineering Conference, vol. 1, Hamamatsu, Shizuoka, Japan, pp. 211-222.

${ }^{[20]}$ Brennen C.E.s., 1994, Hydrodynamics of Pumps, 
New York, Oxford University Press.

${ }^{[21]}$ Valentini D., Pace G., Torre L., Pasini A., and d'Agostino L., 2015, "Influences of the Operating Conditions on the Rotordynamic Forces Acting on a Three-Bladed Inducer Under Forced Whirl Motion", J. Fluids Eng, 137(7), p. 071304. DOI: 10.1115/1.4029887 